\title{
Las barreras de comunicación que tiene \\ el personal de Enfermeria del Hospital \\ General Tacuba del ISSSTE, en México, D.F.
}

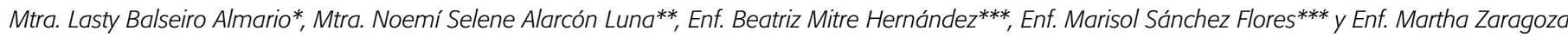
Enriquez***

* Profesor de Carrera Titular "B" de la ENEO-UNAM

**Maestra de Carrera tipo "G" de la Facultad de Enfermería de Nuevo Laredo, de la Universidad Autónoma de Tamaulipas.

***Egresadas de la Escuela de Enfermería del ISSSTE.

El Hospital General Tacuba del ISSSTE es una organización de segundo nivel de atención que se encuentra ubicado en la calle Lago Ontario No. 119 de la Colonia Tacuba. El personal de Enfermería tiene una destacada labor en la atención de los pacientes, para lo cual se cuenta con 387 Enfermeras de las cuales existen: jefes, subjefes, supervisoras, especialistas y auxiliares de Enfermería que cubren las 24 horas de atención a los pacientes. Para ello, el personal de Enfermería se organiza en turnos de trabajo, delegando funciones y actividades, en donde la comunicación tiene una destacada función para hacer posible el lenguaje verbal, corporal y gestual que todas las Enfermeras utilizan para comunicarse con los pacientes. Este estudio realizado en el 2004, tiene por objeto identificar las barreras que tiene el personal de Enfermería en la comunicación para determinar las diversas opciones de solución y así disminuir la problemática del proceso de la comunicación del personal.

Esta es una investigación diagnóstica, observacional, analítica y transversal. El universo son 387 personas, la población de interés para este estudio fueron 272 personas, y la muestra la conformaron el 100\% de la población es decir, las 272 profesionales de Enfermería. Las técnicas e instrumentos utilizados son: fichas de trabajo, la observación, la entrevista y los cuestionarios.

En relación con la variable barreras de la comunicación que tiene el personal de Enfermería en el hospital se pudo determinar que el 92.52\% no tiene ningún temor al comunicarse, el 85.78\% pone atención de lo que se les dice con lo que evitan distorsionar la comunicación y el 73.79\% hablan de manera pausada para no generar problemas de comunicación. En otros datos, el 79.04\% dicen que siempre están buscando la manera de aclarar todo lo que dicen para que se les entienda, el $80.90 \%$ se comunica con mensajes claros que no son distorsionados, el $82.41 \%$ no usan palabras raras cuando se comunican y el $83.15 \%$ siempre son coherentes cuando se comunican. En conclusión, el personal de Enfermería del Hospital General Tacuba no tiene barreras en la comunicación.

PALABRAS CLAVE: Barreras, comunicación, personal de Enfermería.

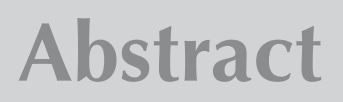

ISSSTE's Tacuba General Hospital is a second-level attention organization located on 119 Lago Ontario Street in Tacuba Colony. The nursing staff has an outstanding job in patients attention, for which there are 387 nurses, ranging from chiefs, sub chiefs, supervisors, specialists, and nursing auxiliaries; all of them cover the 24 hour attention period for the patients. In order to do so, the nursing staff is organized in working shifts, delegating functions and activities, and where communication has an outstanding function to make it possible the verbal, body, and gesture languages that all nurses use for communicating with the patients. This study was carried out in 2004, and aims to identify barriers the nursing staff has for their communication, in order to determine diverse solution options and diminish, thus, the staff communication process problematic. 
This is a diagnostic, observational, analytical, and transversal research. The universe is 387 people, the interest population for the research were 272 persons, and the sample was formed by the 100\% of the population; that is, the 272 nursing professionals. The instruments and techniques used were: working cards, observation, interviews, and questionnaires.

Regarding the variable about nursing staff communication barriers within the hospital, it could be determined that $92.52 \%$ has no fear in communicating, $85.78 \%$ put attention on what one tells them thus avoiding distorting communication and the $73.79 \%$ speak in a paused manner in order not to generate communication problems. In another data, $79.04 \%$ say they are ever looking for way to clarify everything they say so they could be understood; $82.41 \%$ don't use unusual words when communicating and $83.15 \%$ are always coherent when the communicate. Concluding, Tacuba General Hospital nursing staff has no barriers regarding communication.

KEY WORDS: Barriers, communication, nursing staff.

\section{INTRODUCCION}

La comunicación es imprescindible para que se lleve a cabo el contenido funcional del proceso administrativo. Sin la transmisión y recepción de información es imposible planificar, organizar, dirigir y controlar actividades y recursos humanos o materiales. Como ser social, el hombre es dependiente, independiente e interdependiente de los demás individuos del entorno. El único vehículo para relacionarse y establecer vínculos estrechos es la comunicación por medio del lenguaje verbal y no verbal.

Problema de Investigación: El problema de investigación es: ¿Cuáles son las barreras de comunicación que tiene el personal de Enfermería en el Hospital General Tacuba del ISSSTE en México, D.F.?

\section{Objetivos}

El objetivo general del estudio es conocer las barreras de comunicación que tiene el personal de Enfermería en el Hospital general Tacuba del ISSSTE. Los objetivos específicos son: Identificar el tipo de barreras de comunicación que el personal de Enfermería podría tener en el Hospital Tacuba y Proponer diversas medidas que permitan mejorar la comunicación del personal de Enfermería en el Hospital Tacuba.

\section{Justificación}

Esta investigación se justifica porque no existen evidencias fundamentadas de estudios previos y análisis de la variable de Comunicación, en especial las barreras de comunicación del personal de Enfermería que permita identificar la pro- blemática. De igual forma, esta investigación es importante que su estudio se realizara un diagnóstico situacional de la comunicación del personal a fin de conocer claramente la problemática para tomar las decisiones que se juzguen convenientes y solucionar en parte, dicha problemática.

\section{Marco Teórico}

Según Lillian Simms y Cols. la comunicación es la parte central de todo lo que se hace en la administración. La comunicación, o la falta de ella, es uno de los problemas mencionados con más frecuencia de Enfermería. El fin básico de la comunicación es lograr un cambio de nuestro ambiente, en otras personas y en nosotros mismos ${ }^{1}$. Para Elaine L. La Monica, la comunicación representa el punto por medio del cual se relacionan el directivo y los servicios humanos como un sistema. El Directivo se comunicará con eficacia a fin de cumplir verdaderamente su papel. Además, es responsabilidad suya construir y mantener el puente de comunicación, aunque el diseño y estructura participen otros recursos humanos ${ }^{2}$.El proceso de comunicación implica un emisor, un mensaje, un receptor y un puente ${ }^{3}$. La Enfermería como profesión dinámica, no es ajena a la comunicación ya que en su hacer diario se ven involucradas acciones conjuntas con el equipo Inter y multidisciplinario, lo que lleva a la Enfermería a reconocer la comunicación como parte esencial de la vida laboral. Para Ma. José López Montesinos las vías de comunicación son: ascendentes, descendentes, lateral y diagonal ${ }^{4}$. Sin embargo, las barreras de la comunicación pueden interferir en el proceso de la comunicación, 
como el temor y los problemas de los informes escasos, pueden ser porque realmente los subordinados creen que la información no es lo suficientemente importante para comunicarla, o lo que es más frecuente, por temor a las consecuencias de la renovación total al personal superior ${ }^{5}$. Para Ann Marriner el razonamiento defectuoso y los mensajes mal expresados son obstáculos importantes de la comunicación. La falta de claridad y precisión que son el resultado de un vocabulario inadecuado, palabras escasamente escogidas, tópicos, argot, estructuras de frases difíciles, mala organización de ideas y falta de coherencia son muy comunes. El hablar demasiado rápido o lento, las palabras incomprensibles y el no destacar los puntos importantes conduce a la transmisión defectuosa de las ideas. Los informes poco organizados, largos y sin resúmenes también complican el proceso de comunicación. Las palabras significan cosas diferentes para las distintas personas ${ }^{6}$. Independientemente del nivel educativo e intelectual de las personas con que uno intenta comunicarse, sí el material utilizado no es el adecuado, si se utilizan palabras de muchas sílabas o frases largas y complejas es poco probable que ocurra la comprensión de la información.

\section{METOdOLOGÍA}

Es una investigación diagnóstica, observacional, analítica y transversal realizada en 2004. La variable de estudio fué Barreras de la comunicación. La población de interés para este estudio fueron 272 personas, y la muestra la conformaron el 100\% de la población, es decir lo 272 profesionales de Enfermería. El criterio de inclusión fué para todo el personal profesional de Enfermería, el criterio de exclusión fué para el personal no profesional de Enfermería y el de eliminación para aquel personal de Enfermería que no desee participar en la encuesta. Los instrumentos que se utilizaron fueron: fichas de trabajo, la observación, la entrevista y la aplicación de cuestionarios. El principal instrumento de recolección de datos fueron los cuestionarios, el cual tiene preguntas y respuestas estructuradas de tal forma que se pueden medir las barreras de la comunicación del personal de Enfermería, haciendo una pregunta por cada indicador de las barreras de la comunicación. Los datos se procesaron utilizando el sistema de tarjetas simples, de concentración y matrices a fin de obtener la captura, el recuento, clasificación y tabulación en cuadros y gráficas.

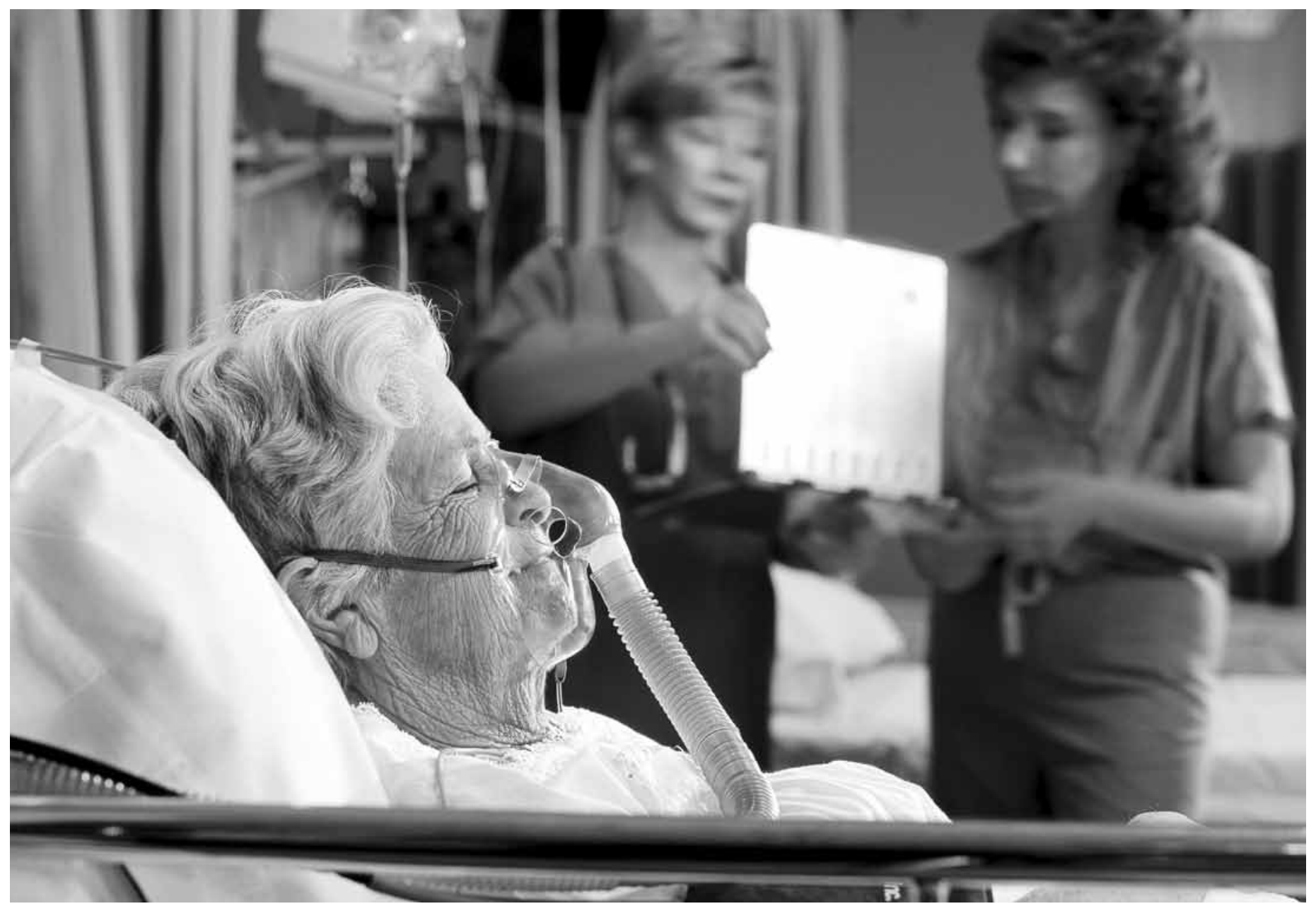




\section{RESULTADOS}

En relación con la variable Barreras de la comunicación que tiene el personal de Enfermería en el Hospital se pudo determinar que el 95.52\% no tiene ningún temor al comunicarse ya que hablan de manera clara, directa y confiable, el 85.78\% pone atención de lo que se les dice con lo que evitan distorsionar la comunicación y el 73.79\% no hablan con rapidez, si no que lo hacen de manera pausada para no generar problemas en la comunicación.

En otros datos se pudo medir que el 79.04\% del personal, siempre están buscando la manera de aclarar todo lo que dicen para que se les entienda y así evitar suposiciones de algún tipo, el 80.90\% se comunican con mensajes claros que no son distorsionados, el $82.41 \%$ no usan palabras raras cuando se comunican porque saben que no les entenderían, y el $83.15 \%$ siempre son coherentes cuando se comunican para que todo el mundo entiendan lo que quieren decir. Otras opiniones del personal entrevistado manifiestan en un $72.29 \%$ que no tienen ninguna desconfianza de las personas que se comunican con ellas porque algo importante tiene que decirles y el $82.78 \%$ saben que la dirección de la comunicación puede ser ascendente, descendente y en varias direcciones con un lenguaje claro y directo.

En relación con el objetivo específico en donde se pedía saber cuáles son las barreras de la comunicación que cree tener el personal de Enfermería se pudo investigar que el $19.86 \%$ consideran que pueden ser por comunicación oral, el 7.87\% que pueden ser por problemas de autoritarismo, el $4.87 \%$ por falta de confianza, $4.86 \%$ por problemas de relaciones humanas, $4.87 \%$ por dificultades en la comunicación y el $4.12 \%$ por falta de formalidad al comunicarse. Llama la atención que para el 14.24\% del personal no existen barreras en la comunicación.

\section{DISCUSIÓN}

Con los resultados anteriormente presentados se puede afirmar que el personal de Enfermería no tiene barreras en la comunicación. Así, globalizando los resultados se puede constatar que el $92.52 \%$ del personal de Enfermería no tiene temor a comunicarse con los demás, ya que atienden perfectamente lo que se les dice y si no es así, lo aclaran, el $85.78 \%$ ponen atención cuando se le habla para evitar distorsionar la comunicación y el 73.79\% habla de forma pausada para que todos entiendan lo que se dice y no haya problemas de comunicación, sin embargo hay un $26.21 \%$ que sí tiene problemas al comunicarse por lo rápido que lo hace. Esto puede ser debido a que en los hospitales hay una gran diversidad de lugares de procedencia del personal que labora en la institución por lo que hablan con rapidez y no se les entiende. El 79.04\% del personal busca la forma de aclarar todo lo que se dice para que les entiendan con claridad y no se hagan suposiciones de ningún tipo aunque un 20.96\% consideran que a veces cuando se comunican quedan suposiciones no aclaradas. Para evitar esto, el $80.90 \%$ opinan que en ningún momento se comunican con mensajes distorsionados, esto puede tener relación con que la mayor parte de las Enfermeras entrevistadas es de nivel operativo y deben tener una comunicación fluida con el personal y los pacientes. En otros datos se encontró que aunque para un $17.59 \%$ existe un lenguaje técnico utilizado en Enfermería, sin embargo el $82.41 \%$ mencionó que nunca utiliza palabras "raras" cuando se comunican, el $83.15 \%$ consideran que es coherente cuando hablan para que entiendan lo que se quiere decir. En relación a si tienen desconfianza en la comunicación, el $72.29 \%$ dicen que no tienen ninguna desconfianza de las personas que se comunican con ellos, aunque hay un $27.71 \%$ que cuentan que a veces si tienen desconfianza y esto puede constituir una barrera importante en al comunicación. El 82.78\% del personal de Enfermería consideran que conocen la dirección de la comunicación y esta no representa ningún problema para ellos. De hecho, las Enfermeras al conocer sus jerarquías conocen plenamente a quien dirigirse cuando se presenta algún problema. Al preguntar específicamente a las Enfermeras cuales creen que serían las barreras que se darían entre el personal, el 19.86\% manifestó que la comunicación oral será la primera, la segunda será por el autoritarismo y la tercera por problemas de relaciones humanas. Esto que todavía no sucede en el Hospital, hay que buscar estrategias para evitar que el personal de Enfermería las padezca.

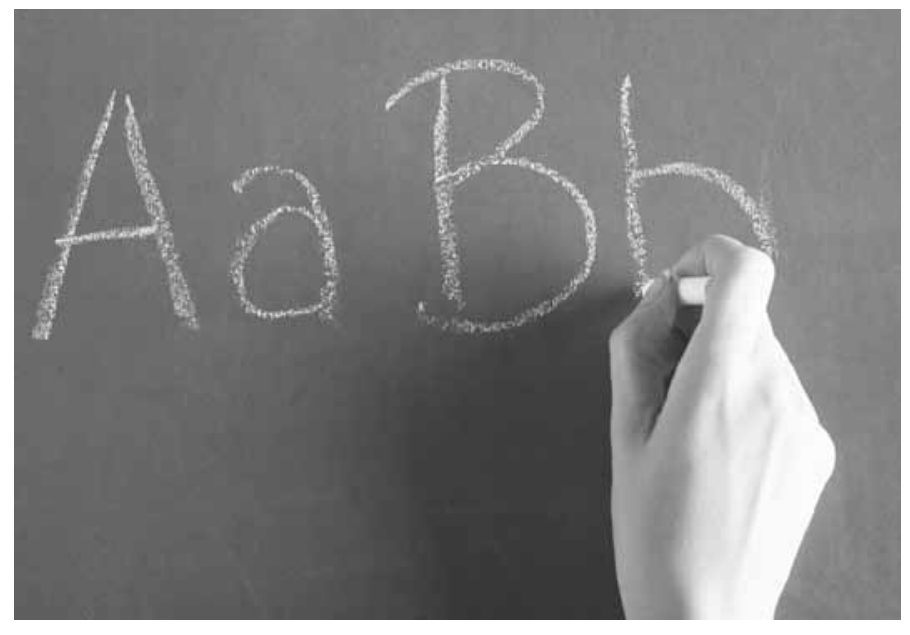




\section{RECOMENDACIONES}

- Proponer a las autoridades que se lleven a cabo diversos cursos de relaciones humanas e interpersonales para mejorar la comunicación entre el personal de Enfermería.

- Motivar al personal de Enfermería, pasante y Enfermeras generales, para que se sigan capacitando y brinden mayor calidad de comunicación y desempeño en su trabajo.

- Sugerir a las autoridades el acercamiento con Enfermeras de diferentes edades para mejorar las relaciones de trabajo y evitar que se generen conflictos laborales.

- Programar cursos de redacción y ortografía para capacitar al personal de Enfermería, de tal manera que puedan redactar mejor las notas de Enfermería y no haya desinformación en la evolución del paciente.

- Invitar al equipo de trabajo a restablecer los canales de comunicación para favorecer el desempeño de sus funciones y así mejorar la atención hacia el paciente.

- $\quad$ Proponer al personal de Enfermería a tener un mejor vocabulario y dicción dentro de su área de trabajo para evitar el deterioro de la comunicación.

- Dar confianza al personal de Enfermería para que se comunique en forma ascendente, descendente, horizontal y diagonal formalmente dentro de su área de trabajo.

- Promover a las autoridades la instalación de pizarrones para la colocación de memorándums y que así exista una mejor comunicación sobre los avisos dentro de la institución.

- Sugerir a las supervisoras que los mensajes e información importante, se elaboren por escrito para evitar fugas de información.

\section{AGRADECIMIENTOS}

A las Enfermeras Beatriz Mitre Hernández, Marisol Sánchez Flores y Martha Zaragoza Enríquez, autoras del estudio, por permitir el análisis y la publicación del artículo, para contribuir al crecimiento profesional de Enfermería.

\section{REFERENCIAS BIBLIOGRÁFICAS}

1. Simms, Lillian et al. Administración de Servicios de Enfermería. Ed. Interamericana. México, 1986, p.244.

2. La Mónica, Elaine. Dirección y administración de Enfermería: Un enfoque práctico. Ed. Mosby Doyma. México, 1995, p. 182.

3. Ibid p. 186.

4. López H.; Ma. José. Administración de Enfermería hoy. Ed. Universidad de Murcia, Madrid, 1998. p. 204.

5. Balseiro, Lasty. Principios de administración. En apoyo a los planes y programas de estudio de Enfermería. Ed.Aztlán. México, 1996, p. 159.

6. Marriner, Ann. Manual para administración de Enfermería. Ed. Interamericana. México, 1993, p.348.

\section{DIRECCIÓN PARA CORRESPONDENCIA}

Mtra. Lasty Balseiro Almario: lastybals@hotmail.com

Mtra. Noemí Selene Alarcón: Lunasalarcon@uat.edu.mx

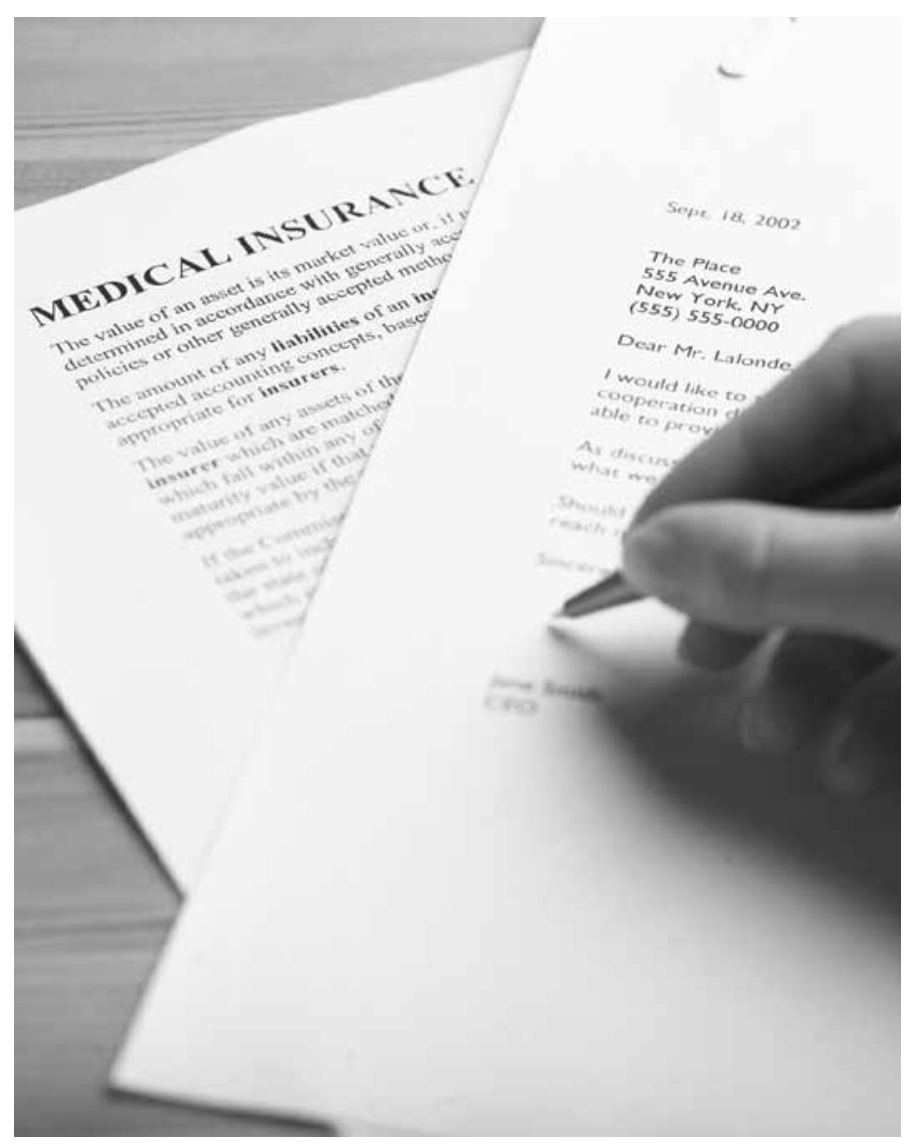

